\title{
EL PENSAR-SE DE LA POBLACIÓN NEGRA Y EL PROBLEMA DEL AGUA EN ESMERALDAS-ECUADOR
}

\author{
Hugo BONE GUANO \\ Universidad Técnica de Esmeraldas "Luis Vargas Torres" (Ecuador) \\ bonehugo.utelvt@gmail.com
}

\begin{abstract}
THE THINK-OF THE BLACK POPULATION AND THE WATER PROBLEM IN ESMERALDASECUADOR
\end{abstract}

Resumen: El estudio del caso del "Agua potable" en Esmeraldas ha sido historia de décadas de colonización interna. La visión del centralismo en Ecuador ha marcado a Esmeraldas como economía de enclave. Los extractivismos en diferentes momentos a partir de las guerras liberales, consolidaron una hegemonía brutal. La provincia ha sido vista como un yacimiento resultando ser una realidad estructural. La negredad es la base de la discriminación que justifica la falta de inversiones y la ausencia de servicios mínimos que humanizan la vida y la convivencia. En 2017 durante varios meses el incumplimiento del acceso al agua ha sido alarmante y dramático; la investigación sobre las respuestas ha concluido en una no respuesta. Seguimos sufriendo restricciones durante largos periodos en las cuales se han perdido ritmos de economía y educación. Las revueltas ciudadanas en otros países andinos por el derecho al agua han tenido resultados, en Esmeraldas se ha Folklorizado. ¿El agua será una herramienta de dominación? asimismo, nos preguntamos si la interseccionalidad de clase y raza son la explicación a las diferencias en los derechos básicos como el agua. Agua que circunda Esmeraldas a modo de fuentes inalcanzables, perdidas en trasiegos de presupuestos extraviados y hurtados a los que contribuyen con sus impuestos. ¿Ser provincia negra nos niega el agua?

Abstract: The study of the "drinking water" case in Esmeraldas has been a history of decades of internal Colonization. The vision of centralism in Ecuador has marked Emeralds as an enclave economy. The extractivisms at different times from the liberal wars, consolidated a brutal hegemony. The province has been seen as a reservoir proving to be a structural Reality. Blackness is the basis of discrimination that justifies the lack of investment and the absence of minimum services that humanize life and living together. In 2017 for several months the breach of access to water has been alarming and dramatic; the investigation into the answers has ended in a non-response. We continue to be constrained for long periods in which economic and educational rhythms have been lost. The citizens ' revolts in other Andean countries for the right to water have had results, in Esmeraldas it has been folklorized. Will water be a tool of domination? We also wonder whether the intersectional class and race are the explanation for differences in basic rights such as Water. Water that surrounds emeralds by way of unreachable sources, lost in the transfer of lost budgets and robbed to those who contribute with their taxes. Is black province denying US water?

Palabras clave: Agua Potable. Discriminación. Negredad. Resistencia. Derechos Básicos Drinking Water. Discrimination. Blackness. Resistance. Basic Rights 
"Una vez que caes en la cuenta de que tu inferioridad es una ficción creada para dominarte, y si no quieres ni asimilarte ni aceptar con resignación la mala suerte de haber nacido donde has nacido, entonces te desprendes. Desprenderse significa que no aceptas las opciones que se te brindan "(Mignolo, 2013: 13).

\section{Introducción}

Este trabajo de campo es un reto académico como estudiante-investigador, y ante el Congreso de Americanistas como plataforma para visualizar y presentar desde el análisis crítico la investigación del agua en Esmeraldas, provincia de la costa norte ecuatoriana. Esta situación del agua potable que afecta la mayor parte de la población, fue motivo para ser parte de lo político en la acción, desde una perspectiva activista de acuerdo con Boaventura, "el científico social no debe diluir su actividad en la de activista, pero tampoco construirla sin relación con el activismo" (De Sousa Santos, 2006: 29).

Partiendo como ciudadano esmeraldeño, con el "deber" de entender y contar lo que ocurre con el agua potable en Esmeraldas, reflexiono el agua potable como problemática emergente, es decir, nuevamente emergente, porque después de largos años no ha dejado de ser el factor común de la población. Este trabajo se refiere, que Esmeraldas no tiene un problema de escasez de agua, de hecho, el sistema del 'Río Esmeraldas' es uno de los más importantes de la costa ecuatoriana, a pesar de, el servicio en la ciudad es entendida por la empresa "EAPA-SAN MATEO" con una mala distribución del recurso hídrico que deja a toda una población sin ella, perdiendo por largos periodos de 15 a 30 días ritmo de economía y educación. En momentos sin agua, el "mercado hídrico" toma protagonismo, desde venta de bidones de agua, aumento del precio de los camiones cisterna y emisión de facturas por servicios inexistentes. Es un escenario de gestión donde hay diversos actores involucrados, existe una relación fantasmal entre políticos y población con discursos y acciones disímiles en relación con la frecuencia de la falta de agua en Esmeraldas, al mismo tiempo, hay más importancia por otros sucesos que por la falta de operaciones técnicas como la captación de agua, conexiones de tuberías, y el manejo de los recursos económicos. Son los principales puntos criticados para una solución a largo plazo, por ejemplo:

La gestión que se hace en Esmeraldas con el agua es completamente defectuosa, porque, aunque se haya hecho inversiones por los Gobiernos para resolver el problema, no se ha logrado; es una problemática histórica (Académico, 2018).

Tradicionalmente al hablar de la política o gestión del agua se aludía a las infraestructuras hidráulicas para las aguas superficiales, fundamentalmente presas y canales (Madurga, 2005: 370), pero en Esmeraldas hay mucho más, por una parte está el incumplimiento constitucional ante los servicios mínimos que humanizan la vida y la convivencia, de acuerdo con la Constitución del Ecuador en el art. 264, numeral 4: "los gobiernos municipales tendrán las siguientes competencias exclusivas sin perjuicio de otras que determine la ley: prestar los servicios públicos de agua potable, alcantarillado, depuración de aguas residuales, manejo de desechos sólidos, actividades de saneamiento ambiental y aquellos que establezca la ley" (Constitución, 2008). Sin embargo, en Esmeraldas sucede todo lo contrario, se admite que es la única ciudad del Ecuador que el Municipio no tiene la competencia del agua.

Asimismo, tenemos el aprovechamiento de los políticos y su promesa eterna, utilizando el agua como un recurso estratégico para obtener puestos de poder, y por otro lado está la población, jugando desde hace algunas décadas en la 'montaña rusa' de sentipensamientos, este término "es empleado para designar aquel hombre que siente y piensa a la vez, el que combina el corazón con la razón” (Díaz, 2015: 185), motivada por la ineficiencia del Gobierno y la falta de agua.

Por más de quince años se ha promovido a nivel mundial la implantación de nuevos arreglos para la prestación de servicios públicos, y en América Latina han incluido un amplio proceso de cambio en el modelo de prestación de agua potable y saneamiento. Ciertamente los cambios realizados en los diversos países de la región han mostrado desiguales niveles de avance y éxito (Corrales, 2002: 48). De avances creo que Esmeraldas sabe muy poco. Mediante el estado del arte descubrí en los antecedentes pocas investigaciones, éstas responden más a la idea del dato, de la historia. De esta manera interpreté que hay una necesidad de un análisis socio-crítico de la realidad en Esmeraldas.

Debido a esto decidí utilizar la etnografía que nutre el presente trabajo, y es "una forma de trabajar que tiene como objetivo buscar el sentido del comportamiento de la gente en relación con los demás" (del Olmo, 2016: 52), utilizando como herramientas fundamentales la observación participante, la entrevista dirigida y abierta, de esta forma entrar en contacto con los diferentes actores formales e informales, y hacer un análisis profundo de la realidad. En mi investigación los colaboradores estaban unidos por una referencia 
en común, sin embargo, se encontró mayor colaboración en la gente con más sentimiento (gente de intercambio social cotidiano con los demás) y menos con la de más pensamiento.

Según Santos, B. "toda ignorancia es ignorante de un cierto saber y todo saber es la superación de una ignorancia particular" (citado en De Sousa Santos, 2006: 79). Este trabajo realizado desde el Sur (metafórico) permitirá poner en discusión el agua potable y el accionar de la población, adjuntándose a las dos formas prácticas de expresar la utopía en Esmeraldas: el rap "SOS Esmeraldas" de Ismael Bernal y el poema "Sed y el Agua" de Antonio Preciado. Emitiendo un análisis crítico que hace intersecciones para un bien común, y amplifica la posibilidad de escuchar las voces esmeraldeñas sin que la política se proteja de lo político:

"El derecho civil se despedaza, tranquilo la bomba se reemplaza, el desespero abraza; el malecón es la coraza, niños, mujeres, ancianos con canecas se desplazan, el tanquero no alcanza, se nota que le llegan gratis hasta la cisterna de su casa, algo raro pasa; ni con el partido del Gobierno el pueblo avanza, no solo se llevaron el dinero a Esmeraldas, le quitaron la esperanza" ... (Bernal).

"Sentida, así como una sola, en masa, resulta una gran sed, casi un tumulto de las que se han venido acumulando; y si en verdad es una sed temible, es mentira que guarde algún encono, justo contra el agua. Por el contrario, hay quienes vuelven el agua turbia, pero esa sed siempre la sueña clara; la viven desviando a sus molinos, pero esa sed ansía reencauzarla; no la dejan correr, pero esa sed quisiera, por fin, desestancarla; o la reporten mal todos los días, es la sed la que alcanza" ... (Preciado).

\section{Método}

Esmeraldas cumpliendo más de 23 años sometida en el centralismo de Ecuador, promoviendo silencio y silenciamientos en una población entera, asimismo, en el 2017 hubo una gran conmoción ante un año muy negativo. Estábamos en pleno auge de restricciones, durante largos periodos perdiendo ritmo de economía y educación hasta marzo 2018. El agua potable tomaba protagonismo en las noticias. Esmeraldas es la ciudad del Sur (metafórico) en Ecuador, sin importar que geográficamente esté en el Norte.

En esta dirección es necesario aclarar que Norte y Sur no son sólo puntos geográficos, administrativos, jurídicos y políticos, sino también lugares de producción de imaginarios sociales y culturales diversos que se mueven en paralelo. Sus cartografías de poder y de saber son redimensionadas desde las tensiones y conflictos que nacen en las fronteras compartidas del imaginario eurocéntrico con Asia, África o América Latina (Martins, 2011: 114).

Ese año fue uno de los puntos de inspiración para surgir desde el contexto de sufrimiento con ilusiones de contribuir y diferenciar los sentipensamientos, alterados por circunstancias que impedían el acceso al agua por semanas consecutivas. Este "trabajo de campo" surgió en mi propio contexto desde la experiencia vivida.

Cuando estaba en el campo, lo más complejo fue cambiar la mirada, porque ésta sucumbe en ocasiones ante un conjunto de ideas y emociones inconscientes que influyen en la investigación. Como investigador autóctono, mi comportamiento y pensamiento tenía que ser diferente, siendo parte de lo investigado tenía que dar un paso fuera del contexto. No obstante, ¿Cómo es posible cambiar la mirada siendo parte del "campo" toda tu vida? Pregunta que no pude contestar, pero, sé que una investigación participativa,

"implica que todas las personas de la comunidad o del grupo donde se está llevando a cabo la actividad, deben involucrarse y llevar el control de todo el proceso de investigación" (Hall, 1983).

Una de las riquezas de la investigación cualitativa es que aparta esa idea de "falsa neutralidad" de la investigación y nos muestra que detrás de un estudio siempre hay un investigador con ideas previas sobre lo que se va a estudiar, que se acerca a esa realidad por unos motivos que pueden ser personales, de orden teórico o prioridades establecidas en las líneas de financiación de diferentes instituciones (Piñeiro, 2015: 84, citado en Gómez, 2017: 40).

Como activista y observador decidí "asumir la subjetividad como una categoría de mayor potencial analítico y emancipador que otras, como conciencia o identidad" (Carrillo, 2006: 91). Asimismo, la subjetividad es asumida como "espacio de las diferencias individuales, de la autonomía y la libertad que se levantan contra formas opresivas que van más allá de la producción y tocan lo personal, lo social y lo cultural" (De Sousa Santos, 1994: 123 citado en Carrillo, 2006: 91).

Ser parte y sufrir la misma problemática del agua ha permitido conseguir un vehículo de expresión, 
esta investigación servirá como plataforma para hacer un análisis profundo de la realidad en Esmeraldas, utilizando "diferentes técnicas de trabajo o herramientas que se desarrollan durante el llamado "trabajo de campo" (del Olmo, 2014: 52). Analizando el contexto como investigador, enfrenté situaciones que me superaban, conduciéndome a diferentes sensaciones con los colaboradores. De esta manera, "esperar" formó parte de las técnicas para obtener las entrevistas con los 'cómplices vivenciales', asimismo, desestimé la posibilidad de varias entrevistas por el desdén y las evasiones que manifestaban algunas personas clave para entender el contexto con relación al agua potable.

La observación participante no es un ejercicio desde otro lugar, necesita nuestra presencia y el registro de lo observado. Es una herramienta de ricos matices porque es un estar sin ser del todo, una situación que se transforma con el paso del tiempo, que tiene un carácter acumulativo y requiere habilidades de flexibilidad negociadora (Gómez, 2017: 42).

Sin embargo, "este tipo de relaciones que establecemos durante la observación participante, también dan lugar a diversos dilemas como, por ejemplo, la definición de nuestro propio rol". (Osuna, 2010 citado en Gómez, 2017: 7). Sin haber definido mi rol en esta investigación llevaré a cabo un análisis en el modo del ser del sujeto en Esmeraldas, la relación que hay entre Esmeraldas y el Estado. Asimismo, el porqué de la invisibilización del problema del agua.

\section{Resultados}

La investigación reveló que en Esmeraldas la "invisibilidad se hace presente, por no tener en cuenta por parte de los grupos hegemónicos, que es una territorialidad con lógicas propias, saben cuáles son sus problemas y demandas en una organización de lucha política colectiva constante" (Valerio, 2013: 2). El trabajo de campo también reveló que,

no ha habido un involucramiento, en realidad lo que ha habido es una especie de críticas aisladas, cada uno hace su propia crítica (Esmeraldeña, 2018).

Críticas con una historicidad en los procesos de la empresa de agua, las relaciones de poder invisibilizadas en los discursos difundidos por la empresa (EAPA) y los medios locales. Se piensa que el agua potable es un problema político, entonces nadie se quiere involucrar para que no lo definan o no lo señalen, eso es todo (Universitaria, 2018), estas personas se distanciaban, se protegían de "decidir sobre los asuntos de la vida en sociedad, de fundar y alterar la legalidad que rige la convivencia humana" (Echeverría cit. Tinajero, 2011: 169). Una realidad que se expresaba a través de una racionalidad diferente:

Bueno, hay puntos de vistas, hay criterios y el uno tiene que ver de cómo se la ha visto a Esmeraldas en la historia, o sea como una provincia de segunda. Saben que aquí en el Ecuador, aunque se quiera esconder hay unas provincias de primera, de segunda, de tercera y hasta puede haber de décimas. Esmeraldas es una de... una provincia realmente que entre estas provincias es una que no se le da mucha importancia (Esmeraldeño, 2017).

Esto es nuevo para unos y viejo para otros, sin importar, ni lo uno ni lo otro, le habían otorgado una escasa relevancia a esta encrucijada en la distribución del recurso hídrico de la provincia. Todas las personas que colaboraron conmigo estaban haciendo política, que "tiene la función de garantizar la institucionalidad de la vida en sociedad" (Eceheverría cit. en Barrios, 2012: 45). Sin embargo, fue axioma de cómo la política se protegía de lo político, se protegía de esta "dimensión ontológica o transhistórica de la vida social, fundada en la idea de que la libertad humana es la posibilidad de la construcción colectiva de un futuro diferente" (Echeverría, 2014: 179 citado en Peña, 2014). Pocas personas consideraban la investigación como vehículo de expresión de la descolonización, otros, la consideraban una vía para la visibilidad, enviándome como mensajero-mediador.

Sin embargo, palpar gente que se movía de su zona de confort para encaminarme a puntos estratégicos para la investigación es una de las formas de ejercer la condición ciudadana. "En este sentido, se es ciudadano cuando irrumpe en la esfera pública para hacer visible la falta de derechos o la necesidad de nuevos derechos" (Mata, 2009: 31). La falta de injerencia y relaciones de la población y los políticos permitió que el uso del agua sea manipulado de forma estratégica para la economía de unos pocos:

Podemos decir, podemos opinar lo que sea, y podemos sabernos toda la parte técnica, pero si no hay una actitud diferente a lo que pasa.... El tema sólo quedará para la anécdota y conversación (Catedrático, 2017). 
Mi rol como investigador fue cambiando paralelamente con los colaboradores, "el que observa el día uno es otra persona el día veinte, pero al mismo tiempo los observados se han ido modificando por su propio crecimiento y la observación. (Gómez, 2017: 42). Me permitió salir de ese bloqueo o ceguera causada por esa "mano invisible", que "es siempre una mano cargada en un solo sentido que condiciona la sociedad civil" (Echeverría, citado en Fuentes, 2014: 178).

De esta forma, encontré una magna colaboración en la población con más sentimiento, puedo decir, que se creó un espacio de "reconocimiento de necesidades e intereses y un ejercicio de análisis acerca de los poderes que niegan la posibilidad de satisfacerlas o hacerlas realidad" (Mata, 2009: 31). La disertación de los colaboradores permitió reconocer e interpretar el modo de ser del sujeto en Esmeraldas, dejando un amplio tema que une a la población de una u otra forma en esta tragicomedia.

\section{El "Upyana yaku" expresada en una "sut'u” (Discusión)}

Me es imposible aceptar que Esmeraldas tenga dificultades para lograr desprenderse del "centro", sin embargo, creo entender que "el Tercer Mundo no fue inventado por la gente que habita en el Tercer Mundo, sino por hombres e instituciones, lenguas y categorías de pensamiento del Primer Mundo" (Mignolo 2013: 13). Esmeraldas como construcción ficticia no fue inventada por los esmeraldeños, fue inventada por un "centro". De acuerdo con Durkheim "la inteligibilidad de los hechos legitima la razón de ser de la ciencia, que aspira a su comprensión y a sustentar la intervención del hombre en dicha realidad" (cit. en Geneyro, 1991: 26). Entonces, pienso que, a raíz del activismo como acción prioritaria, los esmeraldeños tendrán la capacidad de intervenir y presionar desde el "centro" como "periferia". De tal manera que esta "entrada en "el campo" parte del activismo ético, que en esta ocasión no permitimos dejar pasar, como silentes, mansos" (Gómez, 2016: 84).

Los esmeraldeños hemos estado re-conocidos por el Estado como afrodescendientes, sin embargo, el pueblo no se denomina así... ¿Por qué denominar a nivel teórico en la Constitución a un pueblo que no se denomina así? Según Arendt "el problema radica en que las mejores "condiciones sociales" son aquellas bajo la que es posible perder la propia identidad" (Arendt, Cruz y Novales, 1993: 238). ¿Dónde está el derecho a la identidad de Esmeraldas?

"Los ciudadanos necesitamos reconocernos a nosotros mismos y a los demás, necesitamos, en definitiva, un tiempo común que funcione a modo de argamasa que permita que la sociedad no se disuelva" (Blázquez y Piñeiro 2014: 42 citado en Gómez, 2016: 87).

No se ha dado el tiempo de argamasa porque Ecuador convive en dos términos, una es la teoría afrodescendiente, de esta hablan los políticos, la Constitución; pero, los negros es otra cosa, parece que ellos hablan de una gente y la que vive luego es otra. ¿Cuál es la diferencia?, ¿Dónde está el pueblo negro?, se legisla una Constitución admirable y ponen nombre como la "Democracia Intercultural" pero ¿por qué las poblaciones negras están en una situación de extrema pobreza en relación con el resto del país? y ¿por qué en Esmeraldas el agua es una promesa eterna?

El Agua pasó a constituirse en uno de los bienes de consumo esenciales que los individuos y las familias deben garantizarse para sobrevivir. Desafortunadamente, el reconocimiento formal del derecho al agua, que ha sido muy apropiadamente adoptado como un derecho humano fundamental por las Naciones Unidas (UN, 2002 cit. en Castro, Kloster y Torregrosa, 2004: 353), no garantiza en Esmeraldas un acceso regular a dicho recurso.

Sin importar que, en los elementos constitutivos y principios fundamentales del Estado ecuatoriano, establezca en el artículo 3 como deber primordial del Estado (entre otros) el "Garantizar, sin discriminación alguna, el efectivo goce de los derechos humanos, establecidos en la Constitución y en los convenios y más instrumentos internacionales vigentes, en especial la educación, la salud, la alimentación, la seguridad social y el agua para todos sus habitantes" (Acosta y Martínez, 2010: 148).

La Constitución no se circunscribió exclusivamente en impedir la privatización del agua, sino que fue explícita al propiciar la "desprivatización" del agua, incluyendo la redistribución del líquido vital (Acosta y Martínez, 2010: 16). La Constitución nos propone un estado de convivencia, sin embargo, el "campo" dice lo contrario. A inicios del 2018 entrevistando en la universidad "más alta" de Esmeraldas, un académico responde:

el acceso al agua es un problema que resulta sorprendente en una ciudad que tiene uno de los ríos más caudalosos que llega al océano pacífico, y yo creo que es un problema de voluntad política de Estado.

Aún en Esmeraldas hay esa promesa eterna, sin importar que el agua haya pasado a ser un derecho humano fundamental. Todas las promesas de agua se quedaron como "materia en bruto" como diría Bobbio 
(1996) "es precisamente de esta "materia en bruto", y no de aquello que ha sido concebido como "alto y noble", que tenemos que hablar; o, si se quiere, del contraste entre lo que se prometió y lo que efectivamente se hizo.

Después de los resultados del campo me pregunté, ¿Por qué Esmeraldas está en una situación de extrema pobreza en relación con el resto del país?, ¿Será el desconocimiento del Estado sobre la población negra? Por esta razón, conociendo que en la actualidad:

El gobierno de las leyes hoy celebra su triunfo en la democracia. ¿Qué es la democracia si no un conjunto de reglas (las llamadas reglas del juego) para la solución de los conflictos sin derramamientos de sangre?, y ¿en qué consiste el buen gobierno democrático si no, ante todo, en el respeto riguroso de estas reglas? En lo personal no tengo dudas sobre las respuestas a estas preguntas, y precisamente porque no las tengo, puedo concluir tranquilamente que la democracia es el gobierno de las leyes por excelencia (Santillán y Bobbio, 2014).

Conociendo que Esmeraldas es parte de Ecuador, un país denominado democrático y que "cada grupo social necesita tomar decisiones que vinculan a todos los miembros del mismo con el objeto de velar por la propia supervivencia. Ya sea interna o externa" (Bobbio, 1990). Entre las promesas que la democracia no ha podido cumplir se encuentra Esmeraldas, entonces, ¿por qué tantos años Esmeraldas sin tomar sus propias decisiones?, y a raíz de ésta ¿Por qué teniendo los mismos derechos se dan estos hechos con el Agua potable? Desde las teorías decoloniales se puede explicar un poco de por qué Esmeraldas está construida y nombrada por el "centro". Sin embargo, las principales críticas a estas preguntas se centran en el discurso de un esmeraldeño, como cimarrón de la palabra:

Si usted me pregunta cuál es la principal causa, respondo, es de carácter ciudadano. Si la ciudadanía reacciona ante un derecho constitucional irrenunciable, a un derecho a la salud irrenunciable y un hecho a la provisión de agua, es posible que no se hubiera prolongado este problema casi en toda la existencia de la ciudad de Esmeraldas.

En la perspectiva de Mata, P. "un gran número de los obstáculos que perciben para la práctica y el aprendizaje ciudadano constituyen principios básicos de este modelo; entre ellos destaca una fuerte tendencia al individualismo y la consecuente desvalorización de la participación y de lo comunitario" (BallesterosVelázquez, Mata-Benito y Valderrama, 2017: 59):

Los problemas de Esmeraldas van a continuar. Y van continuar por un buen rato a menos que los esmeraldeños levantemos la cabeza y digamos: ¡basta, aquí hay que hacer esto!

De esta manera se expresaba una ciudadana, sentada en una acera con unos amigos después de ver una marcha por la Paz en las calles de Esmeraldas. "el ejercicio de una ciudadanía transformadora parte de una insatisfacción básica con el estado de las cosas y un deseo de cambio" (Mata, P. 2011: 312), los esmeraldeños en sus discursos muestran insatisfacciones, pero siempre estamos esperando que otros hagan, ¿nos faltará motivación para actuar, para transformar esta falta de convicción por el pueblo?

Cuando tú estás en un grupo donde la tendencia natural es esperar a que sea otra persona la que lo haga, si esa otra persona, nunca, digamos siempre responde a esas expectativas, es la que tira del grupo, pues entonces de alguna manera está impidiendo que, que pueda haber otra salida diferente (Historia de vida) (Ballesteros-Velázquez, Mata-Benito y Valderrama, 2017: 64)

La comunidad vive de espaldas a los problemas que le atañen. Si una comunidad da la espalda a sus instituciones o si las autoridades les dan la espalda a los mandantes, eso es lo que ocurre (Palabras de un vocero del comité de vigilancia del agua potable).

Todas estas cuestiones nos llevan a la necesidad de examinar los distintos mecanismos de participación existentes en la actualidad con el fin de poder comprobar su potencia en términos de inclusividad, influencia e intensidad, dimensiones que determinan en gran medida el carácter innovador de unas u otras figuras (Francés, García García y Santacreu, 2007: 25).

Sin embargo, explicando desde las teorías decoloniales, creo que la participación ciudadana no tendrá mucha determinación mientras Esmeraldas dependa de la ontología que le desprende el "centro". Ontológicamente Esmeraldas no es inferior al "centro", el no tener poder del discurso la hace inferior, "aquí, «discurso» se utiliza en el amplio sentido de «acontecimiento comunicativo»" (Van Dijk, 2003: 146).

No se trata de ilustrar la vida social de las personas en un espacio localizado, "sino de rescatar la manera en que actores dispersos espacialmente, aunque unidos por un marco de referencia común -en este 
caso, la crisis hídrica-, actúen más allá de los límites de una supuesta comunidad local de pertenencia" (Koberwein, 2015: 144). Precisamente esa falta de unión no es sino una representación ante un Estado que pretende homogeneizar pero que sigue sin entender qué es ser comunidad negra y su relación con el aguaterritorio-identidad.

Esto no cambiará hasta que los esmeraldeños y esmeraldeñas nos pensemos a nosotros y nosotras, y no a los "otros", y comencemos a nombrarnos, pensarnos como periferia, como el Sur que somos, aunque geográficamente nos ubiquemos en el norte.

El Sur global no es entonces un concepto geográfico, aun cuando la gran mayoría de estas poblaciones vive en países del hemisferio Sur. Es más bien una metáfora del sufrimiento humano causado por el capitalismo y el colonialismo a escala global y de la resistencia para superarlo o minimizarlo (De Sousa Santos, 2010: 43).

Esmeraldas debe nombrarse por sí misma, no debe dejar que la nombren, debe de eliminar esa construcción ficticia inventada por el "centro". En este sentido preguntaba Spivak (1998: 27): “ipuede hablar el subalterno?" Creo que el subalterno únicamente podrá hablar cuando comience a pensar-se, es decir, que comience a reconcocerse y entederse desde sus propios códigos, "pensar el Sur como si no hubiese Norte, pensar la mujer como si no hubiese hombre, pensar el esclavo como si no hubiese señor" (De Sousa Santos, 2006: 74). Pensando desde el Sur podemos tener el poder del discurso. Entonces me pregunto ¿quién inventó al otro, habrá sido el propio esmeraldeño en el proceso de construirse a sí mismo? Por eso de acuerdo con Boaventura, "en mi opinión, no necesitamos alternativas, sino un pensamiento alternativo de alternativas" (De Sousa Santos, 2010: 44).

\section{Conclusiones}

De forma ¡brutal! Este trabajo emergió en un campo donde hay un problema que no está problematizado, que no está discutido y sobre todo que la Academia no ha atendido:

Entonces yo digo, la universidad no está cumpliendo tampoco su papel, o sea no quiero... hago la crítica para proponer algo, jla Universidad ha perdido el rumbo! (Representante Estudiantil, 2018).

Los políticos no le hacen caso a la Academia, porque los académicos tenemos una manera de mirar las cosas, tenemos esa manera de pensar las cosas, y si esa manera de pensar de los intelectuales o de los académicos no cuadra con el pensamiento, con los intereses políticos de los que están en la gestión, simplemente no hacen caso (Antropólogo, 2018).

La Academia se propone como un lugar de debate, de pensamiento crítico, y con perfil ideológico marxista, sin embargo, ¿por qué no se trabaja este tema en la Universidad? Mientras que existe una ínfima literatura sobre este tema, es sorprendente e incomprensible la poca presencia de la Academia en publicaciones y calidad, tomando en cuenta la magnitud del problema.

Investigar el tema del agua en Esmeraldas se vuelve problemático, porque el desprecio al investigador es realmente sorprendente. Se niega información, evaden las entrevistas, y la desconsideración a los tiempos acordados para las entrevistas es un factor común. Ser parte del campo hizo latente mi rol como investigador, las sensaciones de una persona afectada sucumben a la falta de conciencia y a la traición del campo.

Nosotros los esmeraldeños no queremos ser autónomos, queremos que otros hagan, pero es una forma inconsciente, es decir, el problema creo que es la falta de conciencia de que el agua es una herramienta política, una herramienta de dominación.

Concluyo como estudiante-investigador, no juzgo a la calle. De esta forma, este trabajo proyecta servir como plataforma de cambio que permita visualizar y presentar desde el análisis crítico el desprendimiento de la Universidad con la comunidad. Contribuyendo con teorías críticas y en este sentido obtener mentalidades decoloniales. Asimismo, tomar nuestras propias decisiones y condenar el incumplimiento del Estado con relación al servicio de agua potable en Esmeraldas.

\section{Bibliografía}

AA, V. (2014). Taller de investigación cualitativa (Vol. 56). Editorial UNED.

Abeledo, G. G. (2017). Balbinos y Manolitos: Investigación sobre "diglosia convivencial" villa-aldea (del prestigio y privilegio de unos sobre otros). Revista Latina de Sociología, 7(2).

Acosta, A., \& Martínez, E. (2010). El agua, un derecho humano fundamental. Agua; Un derecho humano fundamental, 7-45. 
Arendt, H., Cruz, M., \& Novales, R. G. (1993). La condición humana (Vol. 3). Barcelona: Paidós.

Ballesteros-Velázquez, B., Mata-Benito, P., \& Valderrama, C. E. (2017). Ciudadanía sin escuela. Límites y posibilidades del aprendizaje ciudadano. Revista de Educación Inclusiva, 7(2).

Barrios, M. A. G. (2012). Sobre el concepto de" cultura política" en Bolívar Echeverría. Íconos-Revista de Ciencias Sociales, (43), 33-46.

Bobbio, N., \& Santillán, J. F. F. (1996). El futuro de la democracia.

Carrillo, A. T. (2006). Subjetividad y sujeto: perspectivas para abordar lo social y lo educativo. Revista colombiana de educación, (50), 86-103.

Castro, J. E., Kloster, K., \& Torregrosa, M. L. (2004). Ciudadanía y gobernabilidad en México: el caso de la conflictividad y la participación social en torno a la gestión del agua. El Agua en México Visto desde la Academia, Academia Mexicana de Ciencias, México, DF.

Corrales, M. E. (2002). Gobernabilidad de los servicios de agua potable y saneamiento en América Latina. Caracas, South American Technical Advisory Committee-Global Water Partnership (SAMTACGWP), abril.

De Sousa Santos, B. (2006). Una epistemología del sur: la reinvención del conocimiento y la emancipación social. México: SigloXXI.

De Sousa Santos, B. (2010). Refundación del Estado en América Latina: Perspectivas desde una epistemología del Sur. Plural editores.

Díaz, J. A. R. (2015). Pensamiento crítico en Fals Borda: hacia una filosofía de la educación en perspectiva latinoamericana. Cuadernos de Filosofía Latinoamericana, 36(112), 171-203.

Echeverría, B. (1998). Lo político y la política. UNAM, Instituto de Investigaciones Económicas.

Francés, F., García García, J. T., \& Santacreu, O. 2007). Modelos participativos e innovación democrática local en los municipios valencianos.

Fuentes, D. (2014). Lo político en tiempo de experiencias límite. Capítulo I Crítica a la modernidad y la mundialización capitalistas, 169.

Geneyro, J. C. (1991). La democracia inquieta: E. Durkheim y J. Dewey (Vol. 62). Anthropos Editorial.

Gómez, G. (2016). Medioambientalismo e Interculturalidad. SOS PANADEIRA “Muerte del patrón”. Revista San Gregorio, (15), 80-93.

Hall, B. L. (1983). Investigación participativa, conocimiento popular y poder: una reflexión personal. La investigación participativa en América Latina. Antología, 15-34.

Koberwein, A. (2015). Escasez de agua y apropiación de la tierra en las Sierras Chicas de Córdoba, Argentina. Antípoda. Revista de Antropología y Arqueología, (23).

La invisibilización, d. p. p. d. gtt 22: tierra, agua y territorialidad. Nuevas (¿o no?) estrategias organizacionales colectivas, luchas políticas, rol de la mujer rural y formas productivas soberanas.

Madurga, M. R. L. (2005). Los colores del agua, el agua virtual y los conflictos hídricos. Revista de la Real Academia de Ciencias Exactas, Físicas y Naturales de Madrid, 99, 369-389.

Martins, P. H. (2011). La crítica anti-utilitarista en el Norte y su importancia para el avance del pensamiento poscolonial en las sociedades del Sur. Política \& Sociedades, 10(18), 111-132.

Mata, M. C. (2009). Comunicación comunitaria en pos de la palabra y la visibilidad social.

Spivak, G. C. (1998). ¿Puede hablar el sujeto subalterno? Orbis Tertius, 3(6). 